\title{
Implementing a hybrid SRAM / eDRAM NUCA architecture
}

\author{
Javier Lira*, Carlos Molina ${ }^{\dagger}$, David Brooks ${ }^{\ddagger}$ and Antonio Gonzalez ${ }^{\S}$ \\ *Dept. of Computer Architecture, Universitat Politècnica de Catalunya, 08034 Barcelona, Spain \\ ${ }^{\dagger}$ Dept. of Computer Engineering, Universitat Rovira i Virgili, 43007 Tarragona, Spain \\ $\ddagger$ School of Engineering and Applied Sciences, Harvard University, 02138 Cambridge, MA (USA) \\ $\S$ Intel Barcelona Research Center, Intel Labs - UPC, 08034 Barcelona, Spain \\ javier.lira@ac.upc.edu, carlos.molina@urv.net,dbrooks@eecs.harvard.edu and antonio.gonzalez@intel.com
}

\begin{abstract}
Advances in technology allowed for integrating DRAM-like structures into the chip, called embedded DRAM (eDRAM). This technology has already been successfully implemented in some GPUs and other graphic-intensive SoC, like game consoles. The most recent processor from IBM®, POWER7, is the first general-purpose processor that integrates an eDRAM module on the chip. In this paper, we propose a hybrid cache architecture that exploits the main features of both memory technologies, speed of SRAM and high density of eDRAM. We demonstrate, that due to the high locality found in emerging applications, a high percentage of data that enters to the on-chip last-level cache are not accessed again before they are evicted. Based on that observation, we propose a placement scheme where re-accessed data blocks are stored in fast, but costly in terms of area and power, SRAM banks, while eDRAM banks store data blocks that just arrive to the NUCA cache or were demoted from a SRAM bank. We show that a well-balanced SRAM / eDRAM NUCA cache can achieve similar performance results than using a NUCA cache composed of only SRAM banks, but reduces area by $15 \%$ and power consumed by $10 \%$. Furthermore, we also explore several alternatives to exploit the area reduction we gain by using the hybrid architecture, resulting in an overall performance improvement of $4 \%$.
\end{abstract}

\section{INTRODUCTION}

Technology trends are leading to the use of large on-chip cache memories that typically occupy more than half of the area of the chip. For example, the most recent architecture from Intel $\mathrm{R}$, Nehalem, introduces up to $24 \mathrm{MB}$ shared-L3 cache on the chip, and assigns almost $60 \%$ of the chip area to the cache memory. Cache memories require high bandwidth and fast response times to minimize the number of cycles that a core is stalled due to a memory request. Because of that, traditionally, on-chip cache memories have been implemented with SRAM cells (6T) which are fast and simple. Due to their six-transistor implementation, however, SRAM memories dissipate much more static energy, or leakage, compared to other kind of memories, like DRAM whose cells are implemented with only one transistor and one capacitor. Furthermore, this situation is expected to get worse as the transistor size shrinks in the future technologies. Much research has tackled this problem in SRAM cells by either reducing or removing the power supply to selected cache blocks [6], [8]. However, although leakage currents are reduced, they still persist.
Effectively, DRAM memories dissipate much less static energy than SRAM, however, they are much slower. Actually, DRAM's strongest point is their density, which is more than $3 \mathrm{x}$ compared to SRAM cells. Because of that, DRAM has been typically used to implement off-chip memories, where response time is not so critical. Advances in technology allowed for integrating DRAM-like structures into the chip, called embedded DRAM (eDRAM). This technology has already been successfully implemented in some GPUs and other graphic-intensive SoC, like game consoles. The most recent processor from IBM $R$, POWER7, is the first general-purpose processor that integrates an eDRAM module on the chip [21]. POWER7 uses eDRAM technology to implement a shared 32MByte-L3 cache. By using eDRAM instead of SRAM, POWER7 increases access latency in its third-level cache by few cycles, however, eDRAM provides a roughly $3 \mathrm{x}$ density improvement as well as about $3.5 x$ lower energy consumption than an equivalent SRAM implementation. Prior works in the literature also analysed how to integrate eDRAM technology on the chip efficiently, but eDRAM was always naively integrated as a last-level cache [7], [24]. In this paper, we propose a hybrid cache architecture that exploits the main features of both memory technologies, speed of SRAM and high density of eDRAM.

The hybrid SRAM / eDRAM cache memory we propose in this paper is organized on the chip as a Non-Uniform Cache Architecture (NUCA) design [9]. This kind of cache, which mitigates the effect of increasing on-chip wire delays in cache access latency [2], consist of multiple small banks distributed along the chip that can be accessed independently. Response time in NUCA caches does not only depend on the latency of the actual bank, but also the time required to reach the bank that has the requested data and to send it to the core. So, the NUCA cache latency relies on the physical distance from the requesting core to the bank that has the accessed data block. Furthermore, NUCA caches allow data blocks to be mapped to several banks, thus a data block can migrate from one bank to another without leaving the NUCA cache. For instance, when a data block is accessed by a core, it moves closer to the requesting core in order to minimize access latency for future accesses. In this paper we take advantage of migration movements to share data between SRAM and eDRAM banks.

In this paper we propose a hybrid NUCA cache that 


\begin{tabular}{|l|ccc|}
\hline & Access Time $(\mathbf{n s})$ & Leakage $(\mathbf{m W})$ & Area $(\mathbf{m m} 2)$ \\
\hline SRAM & 0.6631 & 93.264 & 0.4513 \\
eDRAM & 1.4612 & 63.908 & 0.3162 \\
\hline
\end{tabular}

TABLE I: Physical parameters of SRAM and eDRAM memories. The technology assumed is $45 \mathrm{~nm}^{2}$ and their capacity is 64KBytes.

is composed of SRAM banks and eDRAM banks. We demonstrate, that due to the high locality found in emerging applications, a high percentage of data that enters to the on-chip last-level cache are not accessed again before they are replaced. Based on that observation, we propose a placement scheme where re-accessed data blocks are stored in fast, but costly in terms of area and power, SRAM banks, while eDRAM banks store data blocks that just arrive to the NUCA cache or were demoted from a SRAM bank. The effectiveness of this architecture is demonstrated further in this paper. We show that a well-balanced SRAM / eDRAM NUCA cache can achieve similar performance results than using a NUCA cache composed of only SRAM banks, but reducing area by $15 \%$ and power consumed by $10 \%$. Furthermore, we also explore several alternatives to exploit the area reduction we got by using the hybrid architecture, resulting on an overall performance improvement of $4 \%$.

Summarizing, the contributions of this paper are as follows:

1) Implement a hybrid NUCA cache that combines both technologies, SRAM and eDRAM, and make them work cooperatively at the same cache level on the chip.

2) Analyse the behaviour of the proposed hybrid NUCA cache and find the configuration that optimize the trade-off between performance, power and area.

3) Exploit the area and power benefits obtained with the optimal configuration of the hybrid NUCA cache to increase the overall performance.

The remainder of this paper is structured as follows. Section II describes the main characteristics of both technologies, SRAM and eDRAM. Section III lays out the hybrid NUCA cache we proposed in this paper. Section IV presents the experimental method we used, followed by the analysis of the hybrid SRAM / eDRAM architecture that is presented in Section V. In Section VI, we show different alternatives to obtain performance improvements by exploiting area and power reductions. Related work is discussed in Section VII, and concluding remarks are given in Section VIII.

\section{SRAM VS. EDRAM}

SRAM memories are typically used to implement on-chip cache memories because they are faster and easier to integrate than other memories, thus they leverage performance. DRAM memories, however, leverage density. Therefore, this technology has been traditionally used to implement off-chip memories. This section describes the main characteristics of both technologies as well as the role they have in the hybrid architecture we propose in this paper.

SRAM, which is the core storage element used for most register files and cache designs on high-performance microprocessors, is typically implemented with a six-transistor CMOS cell with cross-coupled inverters as storage elements and two pass gates as a combination read/write port. This implementation allows for fast response times and tightly-coupled integration with processing elements which are crucial in a high-performance environment, like register files or low-level caches. On the other hand, as static power dissipated in a circuit relies on the number of transistors of the actual implementation, SRAM caches are significantly affected by leakage currents when they become larger, which is actually the current trend for last-level caches in recently released CMPs.

The memory cell used in DRAM arrays consists of one MOS transistor and a capacitor, where the actual bit is stored. By using such a small memory cells, DRAMs density is about $3 x$ higher than SRAMs. However, 1T1C DRAM memory cell should not be considered for high-performance environment, because a read operation in this memory cell is destructive. The capacitor on the memory cell gets discharged when it is read, so data must be refreshed after each read. This refreshing period stalls the DRAM and cannot be accessed until it is done, so successive accesses to the same bank must queue up and serialize. This increases DRAM memories response time, and thus make them much slower than SRAM. The most straightforward solution is to simply increase the number of independent DRAM banks in order to lower the probability of a conflict. Furthermore, a refresh operation is needed periodically to restore the charge to the capacitor because the leakage current of the storage cell reduces the amount of the stored charge. The refresh operation, which is executed by the sense amplifiers, is vitally important for the correct operation of DRAMs.

The hybrid architecture we propose in this paper is organized as a NUCA cache composed by small banks of both types, SRAM and eDRAM, which are interconnected through an on-chip network. The NUCA organization is further described in Section III. Table I outlines the values of access time, leakage power and area for both kind of memories, SRAM and eDRAM ${ }^{1}$ assuming the same bank size as in the NUCA cache: 64KBytes. We expected SRAM to be a bit faster than DRAM caches, but surprisingly, assuming such a small cache size, SRAM is $2.5 \mathrm{x}$ faster than DRAM. CACTI values also confirm that DRAM cache consumes much less leakage power than SRAM does. Finally, high-density eDRAM cache occupies about two-thirds of the area required by a SRAM cache of the same size.

Based on the features of both technologies, we should build a hybrid NUCA cache that maximizes the number of hits in SRAM banks. Moreover, they should be located close to the cores in order to reduce the overall NUCA latency by

\footnotetext{
${ }^{1}$ SRAM and eDRAM have been modeled with CACTI 5.3. The technology assumed is $45 \mathrm{~nm}^{2}$ and the size of the modeled caches is $64 \mathrm{KBytes}$. More details of the methodology can be found in Section IV.
} 


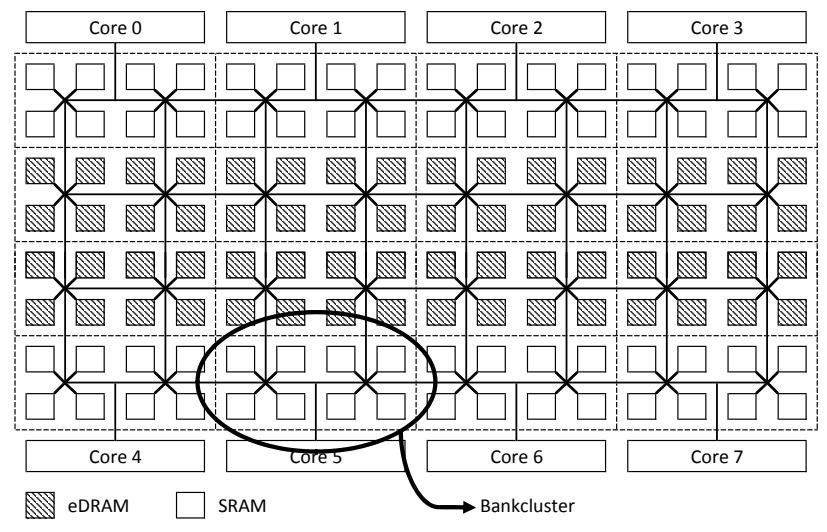

(a) Homogeneous bankclusters

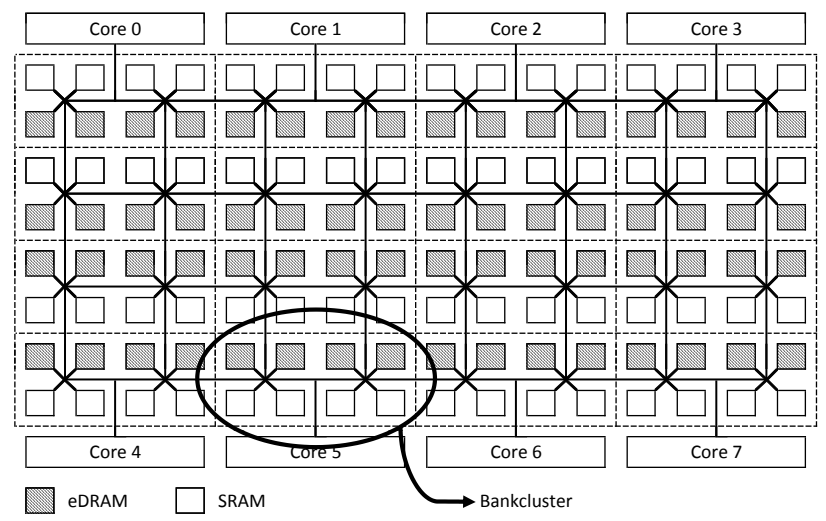

(b) Heterogeneous bankclusters

Fig. 1: Scheme of the proposed NUCA organizations.

minimizing routing delays. With regard to the eDRAM, results on static power and area encourage us to enlarge these banks as much as possible in the NUCA cache. So, we can get significant benefits in terms of power and area with the hybrid architecture.

\section{IMPLEMENTING A HYBRID SRAM/EDRAM NUCA CACHE}

\section{A. Baseline architecture}

We assume a Non-Uniform Cache Architecture (NUCA) L2 cache, derived from Kim et al.'s Dynamic NUCA (D-NUCA) design [9]. Similar to the original proposal we partition the address space across cache banks, which are connected via a 2D mesh interconnection network. As illustrated in Figure 1, the NUCA storage is partitioned into 128 banks. D-NUCA is dynamic in the sense that it allows for migration movements which distribute data blocks among the NUCA banks in order to have the most accessed data close to the cores, and thus reduce access latency for future accesses to the same data. Ideally, a data block could be mapped into any cache bank in order to maximize placement flexibility, however, the overhead of locating a data block may be too large as each bank must be searched, either through a centralized tag store or by broadcasting the tags to all the banks. To address this situation all banks in the NUCA cache are treated as a set-associative structure, and each bank holds one "way" of the set, which are called banksets. Thus, data blocks can only be mapped to one bankset. The NUCA banks that compose a bankset are organized among the NUCA cache in bankclusters (dashed boxes in Figure 1). Each bankcluster consists of a single bank of each bankset. As shown in Figure 1, we propose a NUCA cache 16-way bankset associative that is organized in 16 bankclusters, eight are located close to the cores and the other eight in the center of the NUCA cache. Therefore, a data block has 16 possible placements in the NUCA cache (eight local banks and eight central banks).

The bank where an incoming data block is going to be mapped when it comes from the off-chip memory is statically predetermined based on the lower bits of the data block's address. The LRU data block in this bank would be evicted if it is needed. Once the data block is in the NUCA cache, the migration scheme will determine its optimal position in there. As migration policy, we assume gradual promotion that has been widely used in the literature [9], [4]. It defines that upon a hit in the NUCA cache, the requested data block should move one-step closer to the core that initiate the memory request. With regard to the data search scheme, the baseline D-NUCA design uses partitioned multicast [9], [4]. First, it broadcasts a request to the local bank that is closest to the processor that launched the memory request, and to the eight central banks. If all nine initial requests miss, the request is sent, also in parallel, to the remaining seven banks from the requested data's bankset. Finally, if the request misses all 16 banks, the request would be forwarded to the off-chip memory.

\section{B. The two hybrid approaches}

Figures $1 \mathrm{a}$ and $1 \mathrm{~b}$ show the two different organizations that we propose for the hybrid NUCA cache. Note that both architectures define half of the NUCA banks as SRAM banks, and eDRAM the rest. Based on the main characteristics of SRAM and eDRAM caches, we first propose an intuitive approach that organize all SRAM banks close to the cores and all eDRAM banks in the center of the NUCA cache. We call it homogeneous bankcluster approach and it is illustrated in Figure 1a. This approach does not modify any of the previously described NUCA policies from the baseline D-NUCA cache. Having all SRAM banks concentrated close to the cores, we intend to reduce cache access latency for most accessed data blocks and optimize routing latencies to the SRAM banks. The main drawback of this approach is that by having all eDRAM banks in the center of the NUCA cache it neglects the effect of the migration movements to the shared data. Note that when a data block is simultaneously accessed by two or more cores, it is pulled to different locations by the migration scheme, so it tends to be in the center banks [4].

Figure $1 \mathrm{~b}$ shows the other organization that we propose. It combines SRAM and eDRAM banks within a bankcluster, so we call it heterogeneous bankcluster approach. Compared to the homogeneous bankcluster approach, this organization is not biased to optimizing access latency to the most frequently 


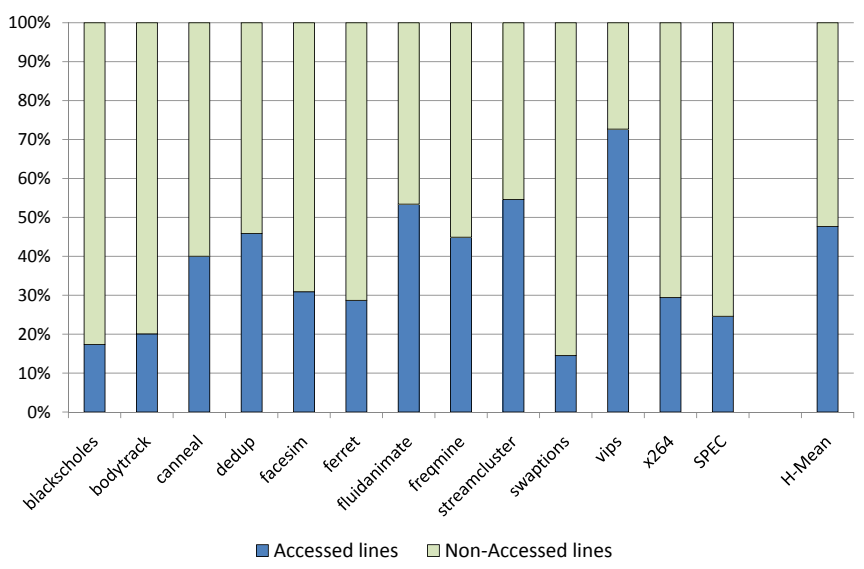

Fig. 2: Percentage of lines that are accessed during their lifetime in the NUCA cache.

accessed data and fairly distributes the fast SRAM banks among the NUCA cache. However, this organization requires SRAM and eDRAM banks to cooperate in order to emphasize the strengths of both technologies and hide their drawbacks.

\section{Placement policy for heterogeneous bankcluster}

In general, when a line is requested by a core, it is stored in the cache memory in order to exploit temporal and spatial locality found in most applications. Cache memory allows for reducing memory response time to the following accesses to the same data and minimizes the number of accesses to the off-chip memory. However, the higher the cache level, the less locality it finds. Figure 2 illustrates the percentage of lines that are accessed during their lifetime in the NUCA cache. We observe that a significant amount of data (more than 50\%) that are stored in the on-chip last-level cache memory are not accessed again during their lifetime in the NUCA cache. It does not mean that they are not accessed at all, but the lower-level cache satisfied these requests due to the high-locality found in the application. Based on this observation, we define a smart placement policy for the heterogeneous bankcluster NUCA organization that works as follows: When a data block enters into the NUCA cache from the off-chip memory, it is located in one of the eDRAM banks (statically predetermined based on the lower bits of its address). Then, if it is accessed again the data block moves to the closest SRAM bank in the bankcluster.

This placement policy assures that SRAM banks store the hottest most frequently accessed data blocks in the NUCA cache, while eDRAM banks have data blocks that were not accessed since they entered to the NUCA cache, and data blocks that were evicted or demoted from SRAM banks. Furthermore, the placement policy in the heterogeneous bankcluster approach introduces the following interesting features:

1) Accessed data blocks always migrate from a SRAM bank to another SRAM bank. It means that once a data block abandons the eDRAM bank to go to a SRAM bank, it will remain in one of the SRAM banks of the

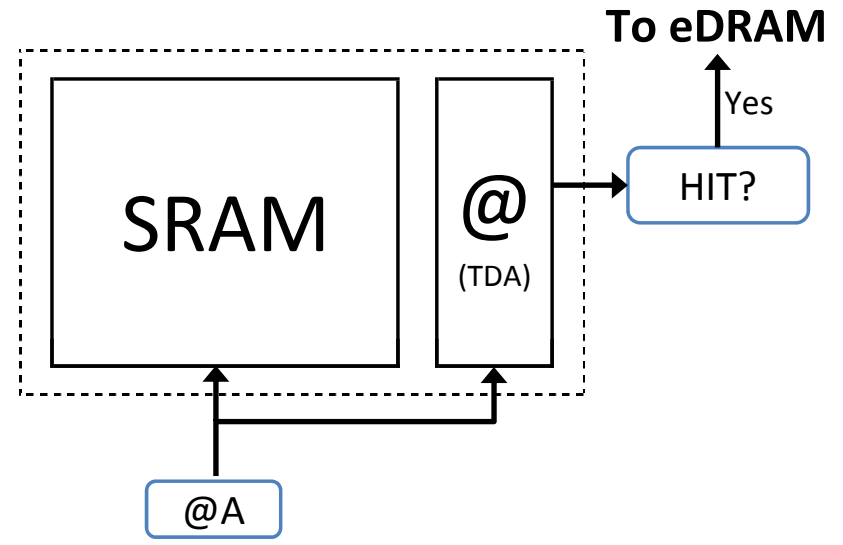

Fig. 3: Scheme of the Tag Directory Array.

NUCA until other more recently used data block takes its place.

2) Gradual promotion stays in the SRAM banks but does not apply for eDRAM banks anymore. There is no communication between eDRAM banks, if there is a hit in one those banks, the requested data block will move towards the closest SRAM bank in the bankcluster.

3) A replacement in a SRAM bank does not provoke an eviction in the NUCA cache. Data blocks that come from the off-chip memory are located in eDRAM banks, so data evictions happen there. SRAM banks, on the other hand, are fed by promoted data blocks from eDRAM banks. Consequently, this provokes data blocks that are evicted from SRAM banks to be demoted to the eDRAM banks instead of being evicted from the NUCA cache.

4) There is a tight relationship between a SRAM bank and an eDRAM bank. Actually, a particular eDRAM bank could be seen as a kind of extra storage for a SRAM bank.

\section{Tag Directory Array (TDA)}

The heterogeneous bankcluster NUCA organization also allows a data block to be mapped to two banks within a bankcluster, one SRAM and one eDRAM. Then, the NUCA cache is 32-way bankset associative in this approach, which is twice the associativity considered with the homogeneous bankcluster approach. As described in Section III-A, increasing the placement flexibility may introduce significant overheads when locating a data block within the NUCA cache that could hurt performance and power of the overall system. Implementing a centralized or distributed tag structure to boost accesses to the NUCA cache in CMP appears to be impractical [4]. Apart from requiring huge hardware overhead, this tag structure could not be quickly accessed by all processors due to wire delays, and more importantly, a separate tag structures would require a complex coherence scheme that updates address location state with block migrations. To address this situation, we propose using the baseline access scheme to find the requested data in the SRAM banks, but also introducing a tag directory array (TDA) 


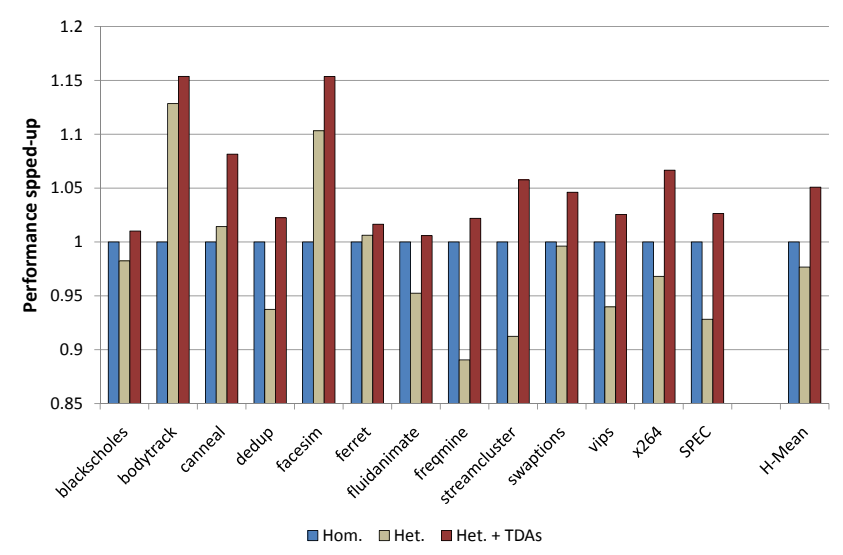

(a) Performance

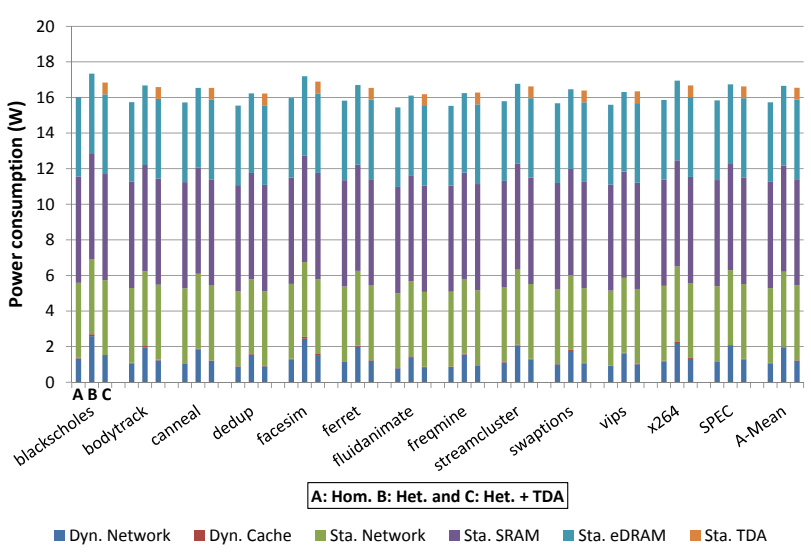

(b) Power consumption

Fig. 4: Results of the three approches described in this section.

per eDRAM bank in order to avoid accessing to any of these banks if they do not have the requested data. As there is no migration between eDRAM banks and each TDA only manages the closest eDRAM bank, this structure does not incur on the overheads previously described for tag structures that manage the whole NUCA cache.

A particular TDA contains the tags of all data blocks that a particular eDRAM bank is storing. In order to provide high-perfomance access, the TDA is implemented using SRAM technology. As illustrated in Figure 3, each TDA is physically located jointly with a SRAM bank. Thus, when a request arrives to the SRAM bank, the joint TDA receives it as well. Then, both structures, the SRAM bank and the TDA, are accessed in parallel, so we prioritise performance at the cost of increasing dynamic power. Finally, if the request hits on the TDA, it is forwarded to the related eDRAM bank, otherwise the request is discarded. By using TDAs, although a data block could be in any of the 32 possible locations within NUCA cache, the implemented lookup algorithm will only need to access up to 17 banks (16 SRAM banks and one eDRAM). This mechanism, however, presents some overheads that should be considered. The most obvious one is the extra hardware required to implement TDAs. For example, assuming an hybrid 4-MByte-SRAM + 4-MByte-eDRAM heterogeneous bankcluster NUCA organization, the total hardware required to implement all TDAs would be 512 KBytes, with each TDA requiring 8 KBytes. The required area and the power dissipated by this structure will be considered when we analyse this mechanism further in this paper. In order to keep each TDA updated, all allocate and deallocate operations in the related eDRAM are synchonized by this structure. Therefore, we maintain correctness in TDAs data at the cost of making these operations a bit slower.

\section{E. Performance and power analysis}

Figures $4 \mathrm{a}$ and $4 \mathrm{~b}$ show how the hybrid approaches that we described in this section behave in terms of performance and power consumption, respectively ${ }^{2}$. Performance results emphasize the necessity of using TDAs with the heterogeneous bankcluster approach. While both approaches, homogeneous and heterogeneous without TDAs, achieve similar performance results, the introduction of TDAs improve performance of the latter by almost $7 \%$. Furthermore, Figure $4 \mathrm{~b}$ illustrates that the power consumed by the heterogeneous bankcluster NUCA organization does not increase when using TDAs, it actually decreases. Because of the bankset-associativity increase, when TDAs are not considered, the heterogeneous bankcluster approach must access more banks to find the requested data block, and thus, it consumes much more dynamic power than the other considered approaches. Although homogeneous bankcluster is the architecture that consumes less power, it does not perform so well. Having all eDRAM banks in the center of the NUCA, this approach is heavily penalized by shared data blocks because they concentrate to these slow banks. In general, we consider the heterogeneous bankcluster approach with TDAs the best choice to be our hybrid NUCA architecture. It performs significantly well assuming both simulated environments, multi-programmed and emerging parallel applications, and it is not constrained by power consumption. Therefore, in the remainder of this paper, we will not evaluate other approaches and will assume we are using the heterogeneous bankcluster approach with TDAs as hybrid NUCA architecture.

\section{EXPERIMENTAL METHODOLOGY}

In order to evaluate the proposed architectures, we used the full-system execution-driven simulator, Simics [11], extended with the GEMS toolset [12]. GEMS provides a detailed memory-system timing model that enabled us to model the presented approaches. Besides, it also integrates a power model based on Orion [20] that we used to evaluate the static and dynamic power consumed by the on-chip network. We have modeled cache memories considered in this paper with CACTI 5.3 [18], which includes support for eDRAM

\footnotetext{
${ }^{2}$ For each approach, we assumed an hybrid 4-MByte-SRAM + 4-MByte-eDRAM NUCA organization. More details of the experimental methodology used are described in Section IV.
} 
caches. It outlines the optimal physical characteristics (e.g. area, access latency and leakage) of the modeled memories. In order to validate CACTI results, we have modeled a real eDRAM macro [16] and found that they are very similar to the actual physical parameters. Thus, we used physical parameters provided by CACTI to evaluate static power consumed and the area required by the memory structures. CACTI has been used to evaluate dynamic power consumption as well, but GEMS support is required in this case to ascertain the dynamic behaviour in the applications. The simulated architecture is structured as a single CMP made up of eight UltraSPARC IIIi homogeneous cores. With regard to the memory hierarchy, each core provides a split first-level cache (data and instructions). The second level of the memory hierarchy is the NUCA cache. In order to maintain coherency along the memory subsystem, we used the MESIF coherence protocol, which is also used in the Intel $\mathbb{R}$ Nehalem processor [13]. The access latencies of the memory structures are based on CACTI models. Table II outlines the most important configuration parameters used in our studies.

\begin{tabular}{|l|l|}
\hline Processors & $8-$ UltraSPARC IIII \\
Frequency & $3 \mathrm{GHz}$ \\
Integration Technology & $45 \mathrm{~nm}$ \\
\hline Block size & 64 bytes \\
L1 Cache (Instr./Data) & 32 KBytes, 2-way \\
L2 Cache (NUCA) & 8 MBytes, 128 Banks \\
\hline L1 Latency & 3 cycles \\
Router Latency & 1 cycle \\
Avg Offchip Latency & 250 cycles \\
\hline
\end{tabular}

TABLE II: Configuration parameters.

We assume two different scenarios: 1) Multi-programmed and 2) Parallel applications. The former executes in parallel a set of eight different SPEC CPU2006 [1] workloads with the reference input. Table III outlines the workloads that make up this scenario. The latter simulates the whole set of applications from the PARSEC v2.0 benchmark suite [5] with the simlarge input data sets. This suite contains 13 programs from many different areas such as image processing, financial analytics, video encoding, computer vision and animation physics, among others.

\begin{tabular}{|cccc|}
\hline $\begin{array}{c}\text { astar } \\
\text { milc }\end{array}$ & $\begin{array}{c}\text { gcc } \\
\text { omnetpp }\end{array}$ & $\begin{array}{c}\text { lbm } \\
\text { perlbench }\end{array}$ & $\begin{array}{c}\text { mcf } \\
\text { soplex }\end{array}$ \\
\hline \multicolumn{4}{|c|}{ Reference input } \\
\hline
\end{tabular}

TABLE III: Multi-programmed scenario.

The method we used for the simulations involved first skipping both the initialization and thread creation phases, and then fast-forwarding while warming all caches for 500 million cycles. Finally, we performed a detailed simulation for 500 million cycles. As performance metric, we use the aggregate number of user instructions committed per cycle, which is proportional to the overall system throughput [22].

\section{EVAluation of THE HYBRID NUCA CACHE}

This section analyses how the hybrid SRAM / eDRAM NUCA architecture presented in Section III behaves in terms of performance, power and area compared to other homogeneous schemes, such as the traditional SRAM NUCA cache, or the same but composed of only eDRAM banks. For the sake of simplicity, all evaluated configurations assume the same NUCA architecture (Figure 1b), so the following parameters do not change along the different configurations: the number of banks in the NUCA cache, on-chip network organization and global NUCA cache size. The configurations evaluated in this section are as follows: 1) all-SRAM NUCA cache, 2) range of hybrid approaches, and 3) all-eDRAM NUCA cache. The former and the latter assume an 8-MByte NUCA cache composed of 128 banks, and behave as described in Section III-A. The hybrid approach also assumes an 8-MByte NUCA cache, but composed of 64 SRAM banks and 64 eDRAM banks. Moreover, in this case we consider seven different configurations by changing the size of the NUCA dedicated to SRAM and eDRAM. This will allow us to find the configuration that better leverages the trade-off between performance, power and area. Table IV outlines the most relevant parameters of the NUCA banks used in the different configurations. In the remainder of the paper we will refer to a X-MByte-SRAM + Y-MByte-eDRAM hybrid NUCA architecture as XS-YD.

\section{A. Performance Analysis}

Figure 5a shows how the hybrid NUCA architecture behaves in terms of performance compared to an all-SRAM-bank NUCA cache. We find that, on average, our hybrid approach can get similar performance results compared to the all-SRAM configuration when considering the proper configuration. Actually, several hybrid configurations, like 5S-3D or 4S-4D, achieve almost $97 \%$ of the performance achieved by all-SRAM. Assuming an all-eDRAM NUCA cache, however, performance is reduced by $13 \%$. Figure $5 \mathrm{~b}$ illustrates the main reason that make our hybrid approach performs so well compared to both homogeneous configurations, all-SRAM and all-eDRAM: the placement policy proposed for the hybrid architecture succeeds in concentrating most of hits in NUCA in the fast SRAM banks, so even dedicating little size to the SRAM banks, most of hits in the NUCA cache happen in the SRAM banks.

By taking into consideration the features of both technologies, SRAM and eDRAM, one could expect that the more size the hybrid NUCA dedicates to SRAM, the better it performs. However, Figure 5a shows that the best performing configurations are those that dedicate about the same size to SRAM and eDRAM banks. As described in Section III, when a data block enters into the NUCA cache it is located into an eDRAM bank. If it is later accessed, then it moves forward to a SRAM bank. This placement decision makes configurations with small eDRAM banks barely effective in terms of performance because, in most cases, data blocks are evicted from the NUCA cache before being accessed for second time, and thus could not move to the SRAM part.

Surprisingly, Figure 5a shows that some hybrid configurations outperform all-SRAM (e.g. bodytrack, streamcluster and vips). This is because our hybrid 


\begin{tabular}{|c|c|c|c|c|c|c|c|c|c|c|c|c|c|c|}
\hline & \multirow[b]{2}{*}{ 諝 } & \multicolumn{4}{|c|}{ SRAM } & \multicolumn{4}{|c|}{ eDRAM } & \multicolumn{5}{|c|}{ TDA } \\
\hline & & 冚 & 弟 & 兽 & $\underset{巳}{\mathbb{Z}}$ & 苞 & 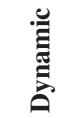 & 莺 & $\underset{Z}{\mathbb{U}}$ & 芯 & 㞯 & 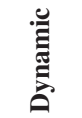 & 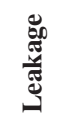 & $\underset{⿱ 乛 龰}{\mathbb{Z}}$ \\
\hline & $\mathrm{KB}$ & ns & $\mathrm{mW}$ & $\mathrm{mW}$ & $\mathrm{mm}^{2}$ & ns & $\mathrm{mW}$ & $\mathrm{mW}$ & $\mathrm{mm}^{2}$ & KB & ns & $\mathrm{mW}$ & $\mathrm{mW}$ & $\mathrm{mm}^{2}$ \\
\hline 1 MByte & 16 & 0.59 & 40.30 & 30.80 & 0.12 & 1.26 & 40.54 & 20.24 & 0.09 & 2 & 0.39 & 22.64 & 1.48 & 0.013 \\
\hline 2 MBytes & 32 & 0.61 & 45.35 & 51.44 & 0.23 & 1.31 & 44.13 & 35.03 & 0.17 & 4 & 0.42 & 23.83 & 2.50 & 0.021 \\
\hline 3 MBytes & 48 & 0.63 & 47.91 & 72.03 & 0.34 & 1.43 & 46.93 & 49.35 & 0.25 & 6 & 0.45 & 25.64 & 3.28 & 0.028 \\
\hline 4 MBytes* & 64 & 0.66 & 49.34 & 93.26 & 0.45 & 1.46 & 49.51 & 63.91 & 0.32 & 8 & 0.49 & 28.83 & 4.35 & 0.036 \\
\hline 5 MBytes & 80 & 0.68 & 52.75 & 114.04 & 0.56 & 1.54 & 53.78 & 76.83 & 0.41 & 10 & 0.53 & 33.51 & 5.39 & 0.042 \\
\hline 6 MBytes & 96 & 0.69 & 55.30 & 135.51 & 0.67 & 1.60 & 56.58 & 91.62 & 0.47 & 12 & 0.56 & 34.03 & 6.60 & 0.053 \\
\hline 7 MBytes & 112 & 0.71 & 58.34 & 156.40 & 0.78 & 1.69 & 59.73 & 105.13 & 0.55 & 14 & 0.57 & 34.55 & 7.61 & 0.068 \\
\hline
\end{tabular}

TABLE IV: Parameters for each configuration got from CACTI models. For example, the shaded parameters are used by the hybrid configuration 2S-6D. *All banks in both homogeneous configurations are like the 4-MByte configuration.

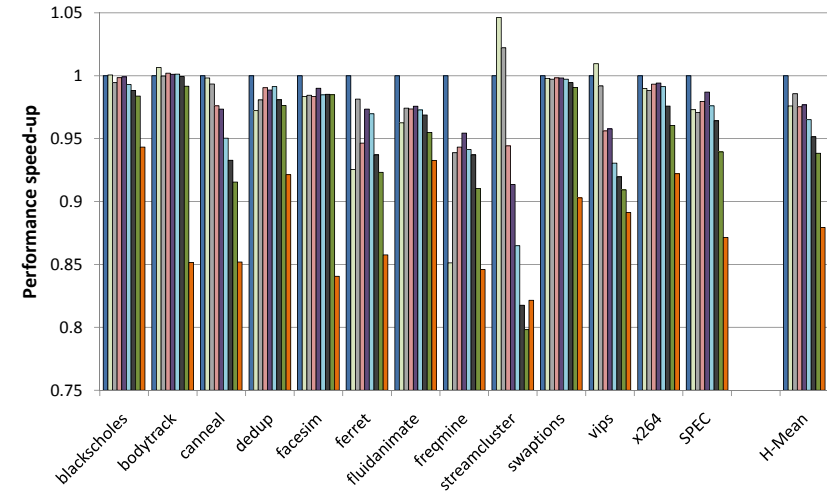

$\square$ All-SRAM $\square 7 S-1 D \quad \square 6 S-2 D \quad \square 5 S-3 D \square 4 S-4 D \quad \square 3 S-5 D=2 S-6 D \quad \square$ 1S-7D $\square$ All-eDRAM

(a) Performance

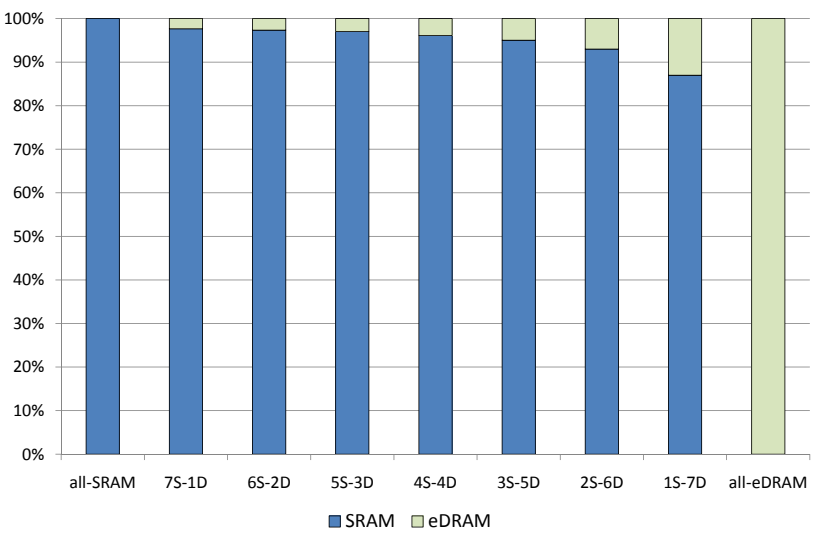

(b) Hit distribution.

Fig. 5: Evaluation of different configurations using the NUCA architecture described in Section III.

architecture increases the bank-set associativity assumed with the homogeneous configurations, then there are more data elegible to be mapped to the SRAM banks. In order to describe better this situation, we will use the following example. A data block that is located in the second row of banks would be in its optimal location for a core in the all-SRAM configuration, however, assuming our hybrid architecture, this data would be in an eDRAM bank, so it could move forward to an SRAM bank in the first row, thus having faster access latency for future accesses.

\section{B. Power and Area Analysis}

Figure 6 a shows the power consumed by each configuration assumed in this analysis. We normalized the power consumption results to the all-SRAM configuration, which is the configuration that dissipates more leakage power, and consequently, consumes more power in general. The major contributor (close to 70\%) of power consumption results is static power. On the other hand, due to the use of the power-efficient eDRAM technology, the all-eDRAM is the least power-consuming configuration, reducing the power consumed by the all-SRAM configuration by $22 \%$. With regard to the hybrid configurations, the less SRAM they use, the less power they consume. In general, their power consumption results range from $3 \%$ (7S-1D) to $18 \%$ (1S-7D) reduction compared to the all-SRAM configuration. Figure $6 \mathrm{a}$ also shows that the overhead associated to TDAs in terms of power consumption is only $2 \%$ assuming the most pessimistic hybrid configuration: 1S-7D. Using TDAs, however, the hybrid architectures prevent accessing all eDRAM banks for each request to the NUCA cache, and thus it prevents increasing dynamic power requirements in these configurations.

With regard to the requirements in terms of die area, Figure $6 \mathrm{~b}$ illustrates the area reduction obtained with the hybrid architecture compared to the all-SRAM configuration. Similar to the trend observed on the power consumption results, the less SRAM the configurations use, the less area they require. The all-eDRAM configuration would occupy less than $70 \%$ of area compared to the all-SRAM. The area reduction of the hybrid architecture ranges from $2 \%$ (7S-1D) to $21 \%$ (1S-7D). In this case, the area overhead introduced by TDAs is not insignificant (up to 10\%), however, as previously shown in this section, this structure is necessary to get performance results similar to the all-SRAM configuration and to reduce dynamic power consumption.

\section{Choosing the best hybrid configuration}

This section shows that the hybrid architecture described in Section III succeeds in combining both technologies, SRAM and eDRAM, in a NUCA cache. We observed that the placement policy assumed benefits these configurations that dedicate about the same size to SRAM and eDRAM banks, 


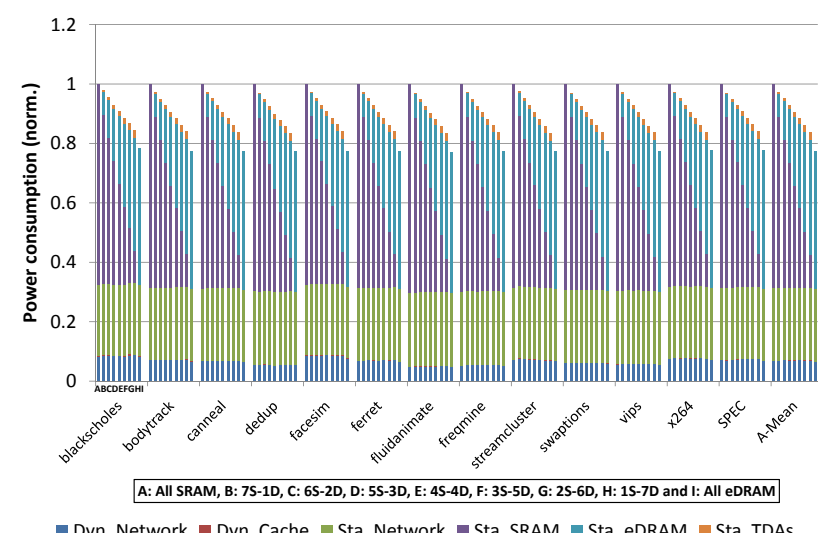

(a) Power consumption

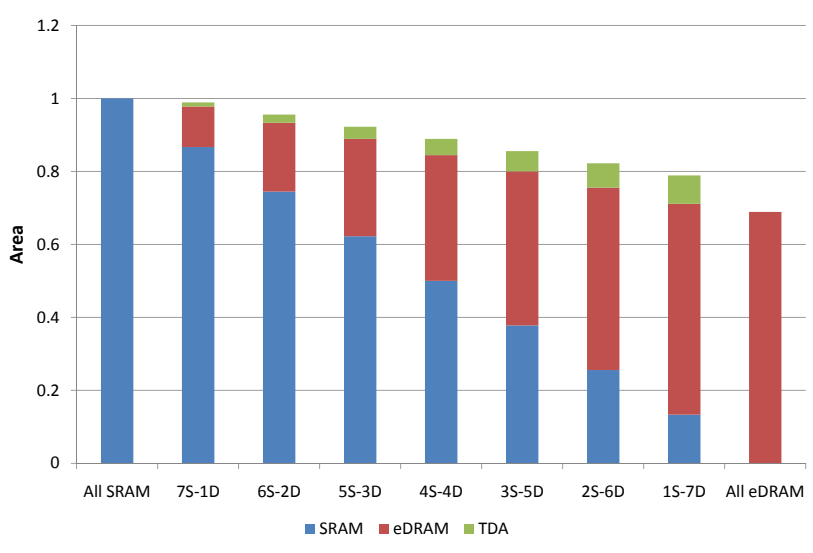

(b) Area

Fig. 6: Power and area results of the configurations assumed.

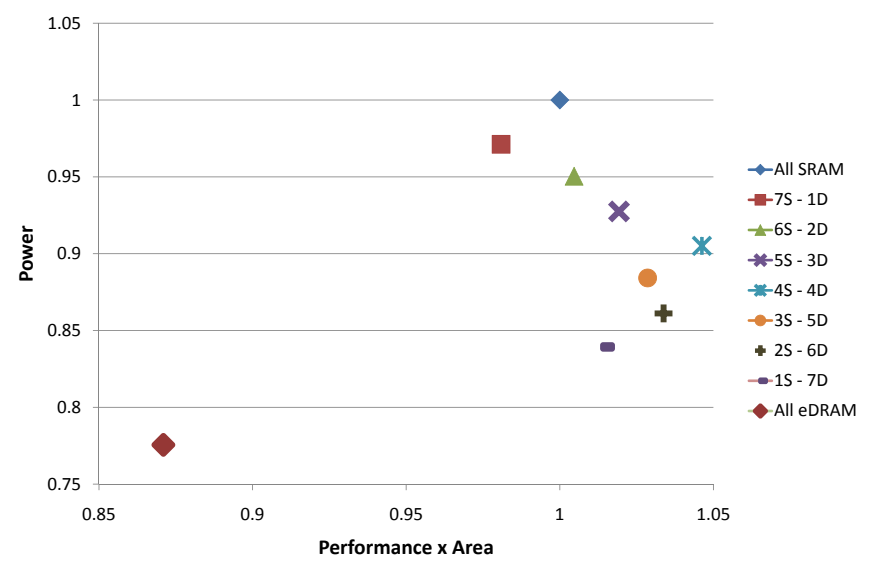

Fig. 7: Trade-off between Power x Performance x Area.

and other imbalanced configurations are not so effective. In order to decide the best hybrid configuration, we could use popular metrics like $\mathrm{ED}$ or $\mathrm{ED}^{2}$ to analyse the performance and power trade-off. However, these metrics does not consider the area required by the configuration, which is very important in our design. So, we have used a recently proposed metric Power $x$ (Performance $x$ Area) [3] that takes into account the three terms of the trade-off to analyse. Assuming the scheme shown in Figure 7, we choose $4 S-4 D$ as the best hybrid configuration. This configuration is neither the best-performing one nor the one that dissipates less power, however, it is the one that leverages better the trade-off between performance, power and area. This is our choice, but in this section we evaluated a wide range of hybrid configurations and showed the main characteristics of all of them. Based on this analysis, architects can choose the hybrid configuration that better fits to their needs, e.g. high performance, low power consumption, or small area.

\section{EXPLOITING ARCHITECTURALS BENEFITS}

Section $\mathrm{V}$ shows that a well-balanced configuration of the proposed hybrid NUCA architecture achieves similar performance results to an architecture composed of only
SRAM banks, but occupies about $15 \%$ less area and dissipates $10 \%$ less power. Architects could manage this significant area reduction to either implement smaller and more power-efficient designs, or re-design architectural structures to improve overall performance. For example, the most recent architecture from Intel $\mathrm{R}$, Nehalem, assigns more than $50 \%$ of the chip area to the last-level cache memory, thus the area reduced of using the proposed hybrid architecture as last-level cache in this processor would be enough to integrate an extra core.

In this section, we evaluate two different scenarios assuming that the remaining transistors are used to increase the last-level cache memory size. Thus, assuming the best hybrid configuration, we increase either the size designated to SRAM banks, or to eDRAM banks. The $15 \%$ area reduction allows for integrating up to 1 MByte extra to the SRAM banks, resulting a 5-MByte-SRAM + 4-MByte-eDRAM hybrid configuration. On the other hand, assuming eDRAM's higher density, architects could manage to re-design the 4S-4D configuration by dedicating up to 6 MBytes to the eDRAM banks. Both configurations, 5S-4D and 4S-6D, occupy almost the same area as a NUCA architecture composed of only SRAM banks.

Figure 8a illustrates how the extended configurations, 5S-4D and $4 \mathrm{~S}-6 \mathrm{D}$, behave in terms of performance compared to the all-SRAM. On average, both configurations outperform the all-SRAM configuration by $4 \%$, and 4 S-4D by $10 \%$. However, we observe that the performance benefits are much higher with memory-intensive parallel applications, like streamcluster and canneal, and with the multi-programmed scenario (mix of SPEC CPU2006 applications). Figure 8a also shows that most of workloads achieve higher performance results with the configuration that provide the largest cache memory (4S-6D), instead of with the one that provides more capacity on fast banks (5S-4D). This demonstrates that reducing the number of accesses to the off-chip memory is more effective in terms of performance than reducing access latency to most frequently accessed data. With regard to the power consumption, Figure $8 \mathrm{~b}$ shows that both configurations, $5 \mathrm{~S}-4 \mathrm{D}$ and $4 \mathrm{~S}-6 \mathrm{D}$, not only occupy the same die area as the all-SRAM configuration, but also dissipate about the same power. 


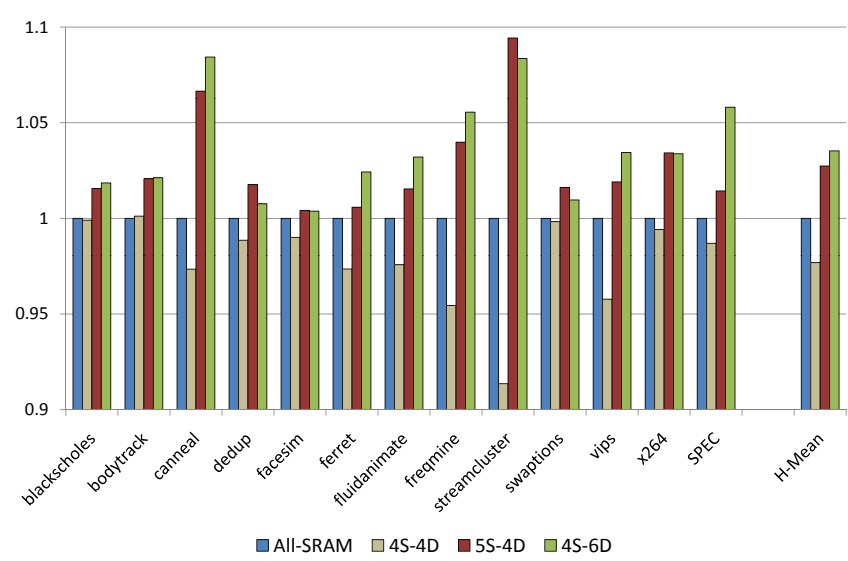

(a) Performance

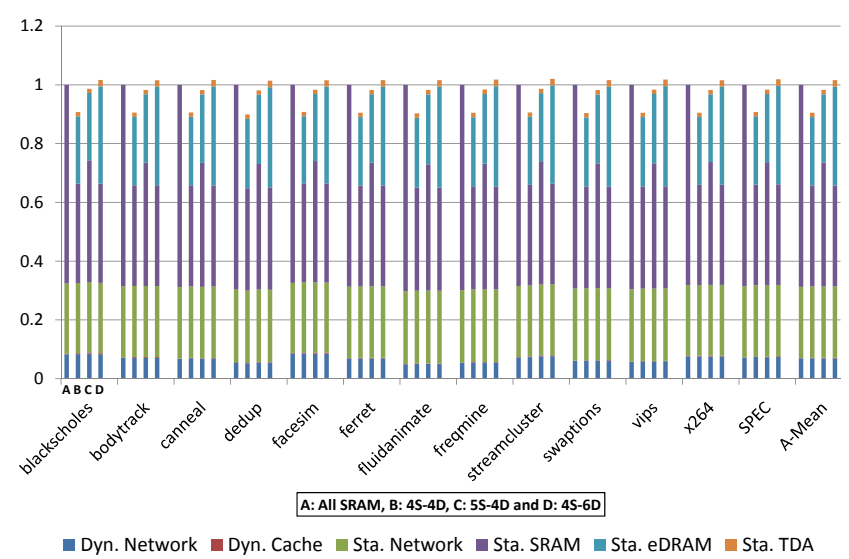

(b) Power

Fig. 8: Performance and power evaluation of our hybrid architecture by assuming the same area as the all-SRAM configuration.

\section{RELATED WORK}

Prior works have proposed hybrid architectures to take advantage of the different features that memories structures offer in the on-chip memory hierarchy. Valero et al. [19] combine both, SRAM and eDRAM, technologies at cell level. They implement a n-way set-associative memory cache with macrocells that consist of one SRAM cell, n-1 eDRAM cells, and a transistor that acts as a bridge to move data from the static cell to the dynamic ones. This design turns out to be very efficient to implement private first-level memory caches. However, it is not so convenient with large shared memory caches where access patterns are not so predictible, and thus significant number of accesses would be to slow eDRAM cells. Our hybrid architecture combines both technologies at bank level, and demonstrate that more than $90 \%$ of hits in the NUCA cache are served by SRAM banks. Wu et al. [23] propose integrating two different technologies (SRAM and eDRAM, MRAM or PCM) at different levels of cache (LHCA), and then, at the same level (RHCA). They split a traditional cache into two regions made up different technologies, and propose a swap scheme to promote data to the fastest region. However, their architecture and placement decisions are based on a private cache scheme, and could hardly be implemented on a shared cache. Another hybrid architecture was proposed by Madan et al. [10]. They propose a 3D chip design consisting of three dies: one contains the processing cores and L1 caches, the second die has the L2 cache which is composed of SRAM banks, and the third die is composed of DRAM banks that act as extra storage to the L2 cache. Furthermore, the placement assumed in this work requires OS support to distribute data blocks among the caches, and consequently to find them in the L2 cache. SRAM and MRAM are also combined to create a L2 hybrid cache in a CMP [17]. This combination tries to solve the problems, in terms of long write latency and high write energy, which MRAM introduces in isolation. Another combination of memory technologies has been recently proposed to be included in main memory [15], [14]. In this hybrid approach, PCM and DRAM are combined in two levels (first level
DRAM and second level PCM) to solve the write endurance problems of the PCM memory and taking profit of its $4 \mathrm{x}$ density compared to DRAM.

\section{CONCLUSIONS}

Cache memories have been typically implemented using SRAM memory structures. They are efficient in terms of latency and are relatively easy to integrate, however, each SRAM cell consists of six transistors, thus these memories are not so efficient in terms of power compared to other alternatives, like DRAM. Recently, advances in technology allowed for integrating DRAM-like memory structures on the chip, called embedded DRAM (eDRAM). These kind of memories provide about $3 \mathrm{x}$ density and dissipate less leakage power than SRAMs, at the cost of not being so fast. IBM $\mathrm{R}$ have already succeeded in integrating eDRAM technology in the on-chip memory cache hierarchy of their latest processor, POWER7. The naive integration of the eDRAM module in POWER7 could be considered the starting point to integrate more sophisticated hybrid cache structures in the near future. Here, we propose a hybrid cache architecture that combines both technologies in the same cache level, and make them work cooperatively. Our architecture, organized as a NUCA cache, uses a smart placement policy that efficiently distributes data blocks among SRAM and eDRAM banks by emphasizing their strengths and hiding their drawbacks. We evaluate a wide range of configurations and observe that a well-balanced hybrid configuration achieves similar performance results to an architecture composed of only SRAM banks, but occupies about $15 \%$ less area and dissipates $10 \%$ less power. Finally, we have analysed different alternatives to take advantage of the area reduction we got by using the hybrid architecture that lead us to get performance benefits up to $10 \%$.

\section{ACKNOWLEDGements}

This work is supported by the Spanish Ministry of Science and Innovation (MCI) and FEDER funds of the EU under the contracts TIN 2010-18368 and TIN 2007-68050-C03-03, the Generalitat de Catalunya under grant 2009SGR1250, and Intel 
Corporation. Javier Lira is supported by the MCI under FPI grant BES-2008-003177.

\section{REFERENCES}

[1] “Spec cpu2006." [Online]. Available: http://www.spec.org/cpu2006

[2] V. Agarwal, M. S. Hrishikesh, S. W. Keckler, and D. Burger, "Clock rate vs. ipc: The end of the road for conventional microprocessors," in Procs. of the 27th International Symposium on Computer Architecture, 2000.

[3] O. Azizi, A. Mahesri, B. C. Lee, S. J. Patel, and M. Horowitz, "Energy-performance tradeoffs in processor architecture and circuit design: A marginal cost analysis," in Procs. of the 37th International Symposium on Computer Architecture, 2010.

[4] B. M. Beckmann and D. A. Wood, "Managing wire delay in large chip-multiprocessor caches," in Procs. of the 37th International Symposium on Microarchitecture, 2004.

[5] C. Bienia, S. Kumar, J. P. Singh, and K. Li, "The parsec benchmark suite: Characterization and architectural implications," in Procs. of the International Conference on Parallel Architectures and Compilation Techniques, 2008.

[6] K. Flautner, N. S. Kim, S. Martin, D. Blaauw, and T. Mudge, "Drowsy caches: Simple techniques for reducing leakage power," in Procs. of the 29th International Symposium on Computer Architecture (ISCA), 2002.

[7] X. Jiang, N. Madan, L. Zhao, M. Upton, R. Iyer, S. Makineni, and D. Newell, "Chop: Adaptive filter-based dram caching for $\mathrm{cmp}$ server platforms," in Procs. of the 16th International Symposium on High-Performance Computer Architecture, 2010.

[8] S. Kaxiras, Z. Hu, and M. Martonosi, "Cache decay: Exploiting generational behavior to reduce cache leakage power," in Procs. of the 28th International Symposium on Computer Architecture (ISCA), 2001.

[9] C. Kim, D. Burger, and S. W. Keckler, "An adaptive, non-uniform cache structure for wire-delay dominated on-chip caches," in Procs. of the 10th Intl. Conf. on Architectural Support for Programming Languages and Operating Systems, 2002.

[10] N. Madan, L. Zhao, N. Muralimanohar, A. Udipi, R. Balasubramonian, R. Iyer, S. Makineni, and D. Newell, "Optimizing communication and capacity in a 3d stacked reconfigurable cache hierarchy," in Procs. of the 15th International Symposium on High-Performance Computer, 2009.

[11] P. S. Magnusson, M. Christensson, J. Eskilson, D. Forsgren, G. Hallberg, J. Högberg, F. Larsson, A. Moestedt, and B. Werner, Simics: A Full System Simulator Platform. Computer, 2002, vol. 35-2, pp. 50-58.

[12] M. M. K. Martin, D. J. Sorin, B. M. Beckmann, M. R. Marty, M. Xu, A. R. Alameldeen, K. E. Moore, M. D. Hill, and D. A. Wood, "Multifacet's general execution-driven multiprocessor simulator (gems) toolset," in Computer Architecture News, 2005.

[13] D. Molka, D. Hackenberg, R. Schone, and M. S. Muller, "Memory performance and cache coherency effects on an intel nehalem multiprocessor system," in Procs. of the International Conference on Parallel Architectures and Compilation Techniques.

[14] M. K. Qureshi, J. Karidis, M. Franceschini, V. Srinivasan, L. Lastras, and B. Abali, "Enhancing lifetime and security of phase change memories via start-gap wear leveling," in Procs. of the 42nd International Symposium on Microarchitecture, 2009.

[15] M. K. Qureshi, V. Srinivasan, and J. A. Rivers, "Scalable high-performance main memory system using phase-change memory technology," in Procs. of the 32th International Symposium on Computer Architecture, 2009.

[16] S. Romanovsky, A. Katoch, A. Achyuthan, C. O'Connell, S. Natarajan, C. Huang, C. Y. Wu, M. J. Wang, P. Chen, and R. Hsieh, "A 500mhz random-access embedded $1 \mathrm{mb}$ dram macro in bulk cmos," in Procs. of the IEEE International Solid-State Circuits Conference (ISSCC), 2008.

[17] G. Sun, X. Dong, Y. Xie, J. Li, and Y. Chen, "A novel 3d stacked mram cache architecture for cmps," in Procs. of the 15th International Symposium on High-Performance Computer, 2009.

[18] S. Thoziyoor, N. Muralimanohar, J. H. Ahn, and N. P. Jouppi, "Cacti 5.1," HP, Tech. Rep., 2008.

[19] A. Valero, J. Sahuquillo, S. Petit, V. Lorente, R. Canal, P. Lopez, and J. Duato, "An hybrid edram/sram macrocell to implement first-level data caches," in Procs. of the 42nd International Symposium on Microarchitecture, 2009.

[20] H. S. Wang, X. Zhu, L. S. Peh, and S. Malik, "Orion: A power-performance simulator for interconnection networks," in Procs. of the 35th International Symposium on Microarchitecture, 2002.
[21] D. Wendel, R. Kalla, R. Cargoni, J. Clables, J. Friedrich, R. Frech, J. Kahle, B. Sinharoy, W. Starke, S. Taylor, S. Weitzel, S. G. Chu, S. Islam, and V. Zyuban, "The implementation of power7: A highly parallel and scalable multi-core high-end server processor," in Procs. of the IEEE International Solid-State Circuits Conference (ISSCC), 2010.

[22] T. F. Wenisch, R. E. Wunderlich, M. Ferdman, A. Ailamaki, B. Falsafi, and J. C. Hoe, "Simflex: Statistical sampling of computer system simulation," IEEE Micro, vol. 26, no. 4, pp. 18-31, 2006.

[23] X. Wu, J. Li, L. Zhang, E. Speight, R. Rajamony, and Y. Xie, "Hybrid cache architecture with disparate memory technologies," in Procs. of the 32th International Symposium on Computer Architecture, 2009.

[24] L. Zhao, R. Iyer, R. Illikkal, and D. Newell, "Exploring dram cache architectures for cmp server platforms," in Procs. of the International Conference on Computer Design, 2007. 